\title{
Modelling the Multifrequency SED of AGN Candidates among the Unidentified EGRET and Fermi Gamma-Ray Sources
}

\author{
Pieter J. Meintjes ${ }^{1}$, Pheneas Nkundabakura ${ }^{2,1}$, Brian van Soelen ${ }^{1}$, Alida Odendaal ${ }^{1}$ \\ ${ }^{1}$ Department of Physics, University of the Free State, P.O. Box 339, Bloemfontein, 9300, South-Africa \\ ${ }^{2}$ Kigali Institute of Education, P.O. Box 5039, Kigali, Rwanda \\ Corresponding author: MeintjPJ@ufs.ac.za
}

\begin{abstract}
Of the 271 sources in the $3^{\text {rd }} E G R E T$ catalogue, 131 were reported as unidentified, i.e. not associated with any particular class of point source in the sky. Since the largest fraction of the EGRET sources were extragalactic, a sample of 13 extragalactic unidentified sources have been selected for multi-wavelength follow-up studies. Five of the selected EGRET sources coincide with gamma-ray flux enhancements seen in the Fermi-LAT data after one year of operation.

In this article, we report the multi-wavelength properties of, among others, the 5 sources detected by Fermi-LAT from our sample of high galactic latitude unidentified EGRET sources. Recent spectroscopic observations with the Southern African Large Telescope (SALT) confirmed one of the unidentified EGRET sources as a possible Seyfert 2 galaxy, or alternatively, a narrow line radio galaxy. The detected gamma-ray emission $\left(E_{\gamma}>30 \mathrm{MeV}\right)$ of the 5 coinciding EGRET/Fermi-LAT sources are fitted with external Compton and Synchrotron Self Compton (SSC) models to investigate the energetics required to produce the EGRET/Fermi gamma-ray flux. In all the models the inclination angle of the jet with respect to the observer is $\theta_{\text {jet }} \approx 60^{\circ}$, between those of Seyfert 1 and Seyfert $2 /$ radio galaxies. These results confirm the possibility of Seyfert and radio galaxies sources are constituting a new class of $\gamma$-ray source in the energy range $E_{\gamma}>$ $30 \mathrm{MeV}$.
\end{abstract}

Keywords: radiation mechanisms: non-thermal - line: identification - techniques: spectroscopic - galaxies: jets BL Lacertae objects.

\section{Introduction}

The Energetic Gamma Ray Telescope Experiment EGRET (30 MeV - $10 \mathrm{GeV})$ provided the highest gamma-ray window on board the Compton GammaRay Observatory (CGRO). EGRET detected 271 gamma-ray sources above $100 \mathrm{MeV}, 92 \%$ of which were blazars. Of the 271 sources detected, 131 remained unidentified, i.e. could not be associated with any specific point source of gamma-ray emission (Hartman et al., 1999). The large number of unidentified EGRET sources above and below the galactic plane inspired a search for possible extra-galactic radio loud Active Galactic Nuclei (AGN) counterparts that could possibly be associated with these unidentified sources. To avoid confusion with possible galactic sources, especially molecular cloud distributions, the search for counterparts was restricted to those unidentified sources at galactic latitudes $|b|>10^{\circ}$.

Source Selection Criteria: The candidate counterparts should be inside the error box associated with the EGRET detection (Hartman et al., 1999), confirmed as extragalactic in the NASA Extragalactic Database (NED), possess a radio brightness above 100
mJy at $8.4 \mathrm{GHz}$ (Sowards-Emmerd, Romani \& Michelson, 2003), exhibit hard spectra with spectral indices $|\alpha|<0.7$ (Sowards-Emmerd, Romani \& Michelson, 2003) and display variability (e.g. Fan, 2005) that may be associated with an inner accretion disc or jet. Based upon these criteria, 13 sources have been selected for further follow-up study (Meintjes \& Nkundababura, 2012; Nkundabakura \& Meintjes, 2012).

Of the 13 candidate sources selected, 5 have confirmed gamma-ray excesses in the Fermi-LAT catalogue containing the first year's observations (Abdo et al., 2010a). The Fermi Large Area Telescope (Fermi$L A T)$ is a pair conversion $\gamma$-ray telescope sensitive to photon energies between $20 \mathrm{MeV}$ and $300 \mathrm{GeV}$. Launched on 11 June 2008, Fermi-LAT started to collect data in August 2008. The data are made available on a daily basis and can be accessed online at the official website of the Fermi Science Support Centre (http://fermi.gsfc.nasa.gov/cgi-bin/ssc /LAT/LATDataQuery.cgi). The results of detections made within the first 24 months of operation were released in June 2011, in the form of the Second Fermi-LAT catalog 
(2FGL; see Nolan et al., 2012) ${ }^{1}$.

Five sources from our previous sample of unidentified EGRET sources have been detected with FermiLAT, namely 2FGL J0727.0-4726, 2FGL J1304.3-4353, 2FGL J1703.2-6217, 2FGL J1709.0-0821 and 2FGL J1815.6-6407, which are counterparts of 3EG J07244713, 3EG J1300-4406, 3EG J1659-6251, 3EG J17090828 and 3EG J1813-6419 respectively. The Fermi-LAT sources that coincided with the EGRET sources are presented as circled crosses in Fig. 1.

The EGRET (30 MeV-10 GeV) gamma-ray spectra of our chosen sample of sources that were observed between April 1991 - October 1995 (cycles 1, 23 and 4 of the mission) have been determined. The photon spectral index distribution is displayed in Fig. 2. The spectral distribution of these unidentified sources corresponds remarkably well with the gamma-ray blazar photon spectral index distribution observed by FermiLAT (Abdo et al., 2010a).

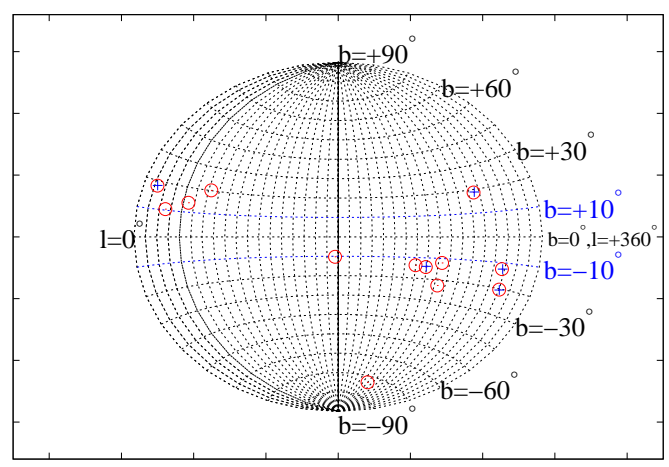

Figure 1: Galactic distribution of the unidentified EGRET sources (circles) and their Fermi-LAT counterparts (crosses).

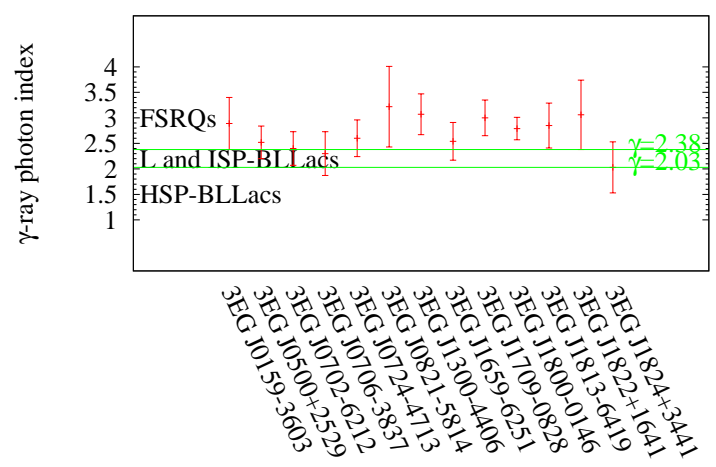

Figure 2: Gamma-ray photon spectral indices of the EGRET sources between $30 \mathrm{MeV}-10 \mathrm{GeV}$.

\footnotetext{
${ }^{1}$ http://fermi.gsfc.nasa.gov/ssc/data/access/lat/2yr_catalog/
}

The gamma-ray spectra of the sample of EGRET sources with Fermi-LAT counterparts were determined. The spectra are presented in Fig. 3. Noticeable is the apparent change in the spectral index between the EGRET and the Fermi-LAT gamma-ray data, which may point to a transition in the gamma-ray production process.

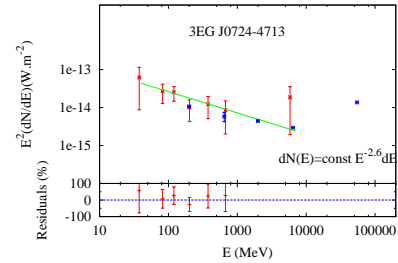

a.) $3 E G$ J0724-4713

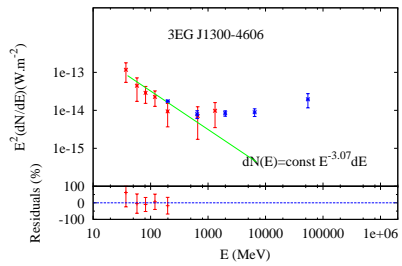

c.) $3 E G J 1300-4406$

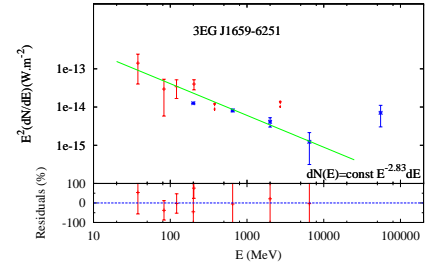

b.) $3 E G J 1659-6251$

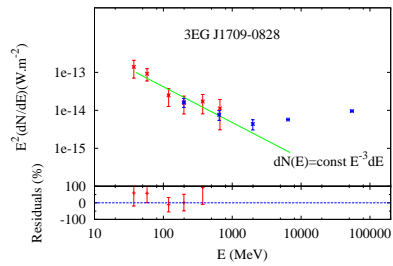

d.) $3 E G ~ J 1709-0828$

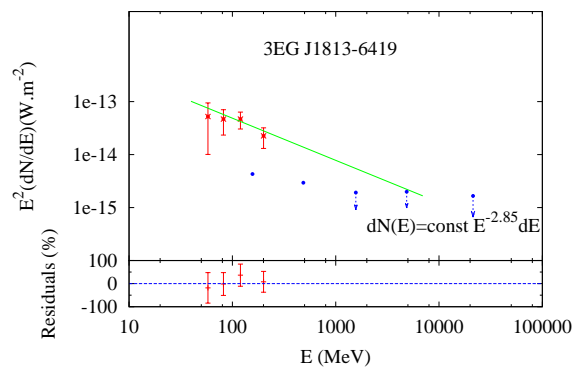

e.) $3 E G J 1813-6419$

Figure 3: The gamma-ray spectra of the EGRET (30 $\mathrm{MeV}-10 \mathrm{GeV})$ and coinciding Fermi-LAT $(20 \mathrm{MeV}$ $300 \mathrm{GeV}$ ) sources.

\section{Gamma-Ray Variability}

The multi-wavelength emission of accretion driven sources like AGN is characterized by very high luminosity, assuming isotropic emission, and variability over several time scales (e.g. Fan, 2005). This is reconciled with the fact that the bulk of the Spectral Energy Distribution (SED) of these sources is produced in a non-homogeneous and variable jet. Gamma-ray flux 
variability has also been confirmed from AGN-blazars (Mattox et al., 1997).

Aperture photometry of the Fermi-LAT data over a time span of 4.6 years (from August 2008 to March 2013) has been performed to investigate possible long term variability. Photons in the energy interval 100 $\mathrm{MeV}$ to $200 \mathrm{GeV}$ were counted in an area with a radius of 1 degree centered on the source and monthly averages were determined. Two of the sources, $2 \mathrm{FGL}$ J1304.3-4353 and 2FGL J1703.2-6217, showed definite signs of variability, quantified in terms of a variability index V (Nolan et al., 2012), which implies that for $\mathrm{V}>41$ there is a $<1 \%$ probability of the source being steady. Although variability was detected in $2 \mathrm{FGL}$ J1304.3-4353 (V = 47) and 2FGL J1703.2-6217 (V = 167) respectively, no periodicity could be detected (see Fig. 4).

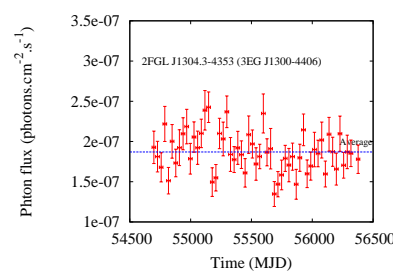

a.) $2 F G L J 1304.3-4353$

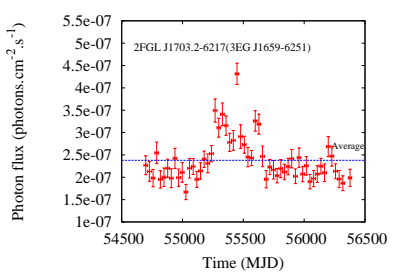

b.) $2 F G L J 1703.2-6217$
Figure 4: The gamma-ray lightcurves of the two Fermi-LAT sources, 2FGL J1304.3-4353 and 2FGL J1703.2-6217, which displayed variability over a period of 4.6 years. The vertical error bars indicate the $68 \%$ confidence level.

\section{Optical Follow-Up Studies}

The optical counterparts of two unidentified EGRET sources have already been identified (Meintjes \& Nkundabakura, 2012; Nkundabakura \& Meintjes, 2012). The spectrum of PKS J0820-5705 (3EG J08215814) resembles that of a FSRQ at redshift $\mathrm{z}=$ 0.06, while the spectrum of PMN J0710-3850 (3EG J0706-3837) shows broad and narrow lines resembling the spectrum of a LINER or Seyfert I galaxy at redshift $\mathrm{z}=0.129$. What distinguishes the spectrum of PKS J0820-5705 from that of a normal radio galaxy is the shallow K4000 depression of only 8.8 $\pm 2.5 \%$, indicating substantial non-thermal activity, while the corresponding value for PMN J0710-3850 is $80 \pm 1 \%$ (Meintjes \& Nkundabakura, 2012; Nkundabakura \& Meintjes, 2012), in agreement with the value expected for a LINER-Seyfert 1 galaxy (e.g. Caccianiga et al., 1999).

Spectroscopic observations of other sources from our sample have been performed with the Southern African Large Telescope (SALT) (see Fig. 5), equipped with the Robert Stobie Spectrograph (RSS), during 2012, in order to determine the redshift and to identify the class of AGN. The spectrum of one of the unidentified EGRET sources, 3EG J 0159-3603, could be determined (see Fig. 6 ), showing distinct narrow emission lines of O II, O III and He II redshifted by $\mathrm{z}=0.35$. The spectrum resembles that of a typical Seyfert 2 galaxy, or alternatively, a narrow line radio galaxy. This implies the possible association of two Seyfert galaxies with the unidentified sources 3EG J0706-3837 and 3EG J 0159-3603 respectively.

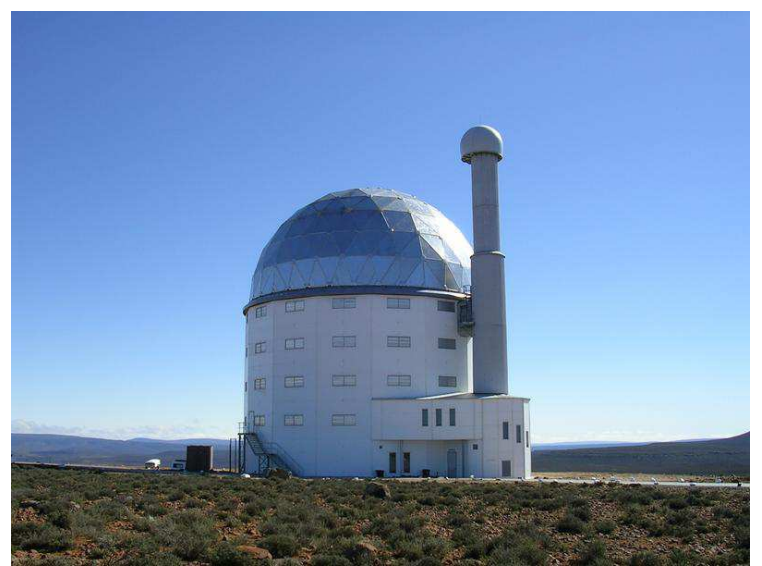

Figure 5: The Southern African Large Telescope (SALT).

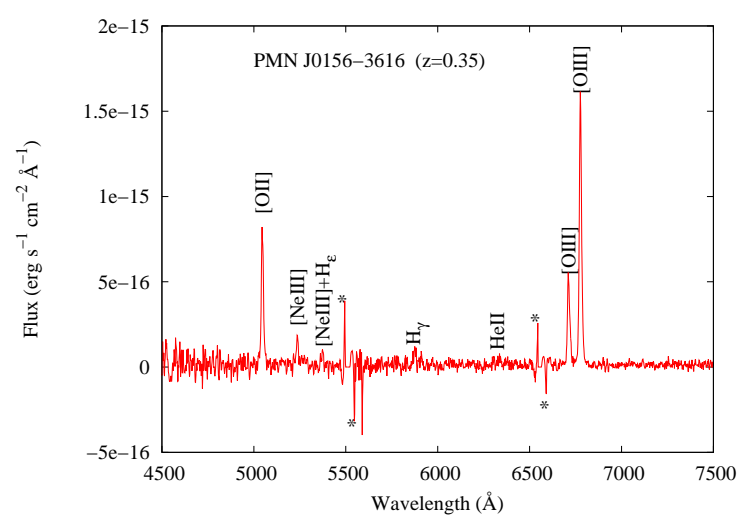

Figure 6: SALT spectrum of the optical counterpart of the unidentified EGRET source 3EG J0159-3603, showing emission lines redshifted by $\mathrm{z}=0.35$

\section{The Spectral Energy Distributions (SED)}

The spectral energy distributions (SEDs) of all the counterparts corresponding to the unidentified EGRET 
sources have been pieced together through multiwavelength archival data as well as multi-wavelength observations from radio to optical, using the Hartebeesthoek Radio Astronomy Observatory (HartRAO) $26 \mathrm{~m}$ telescope, as well as various optical telescopes at the South African Astronomical Observatory (SAAO) in South Africa. Infrared data were obtained from the Two Micron All Sky Survey (2MASS) conducted between June 1997 and February 2001. The SEDs of the sample of EGRET sources and the associated Fermi$L A T$ counterparts are presented in Fig. 7 .

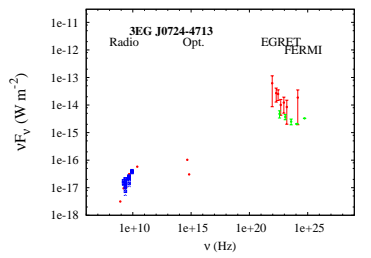

a.) $3 E G J 0724-4713$

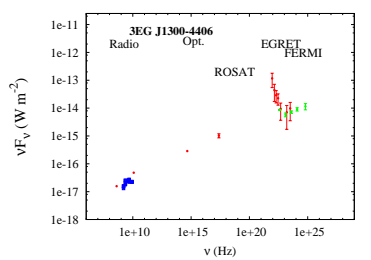

c.) $3 E G J 1300-4406$

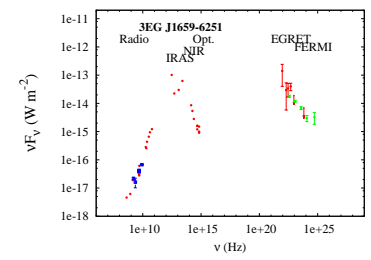

b.) $3 E G J 1659-6251$

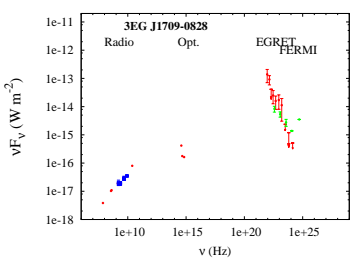

d.) $3 E G$ J1709-0828

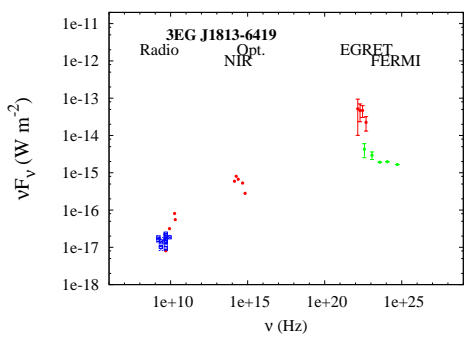

e.) 3EG J1813-6419

Figure 7: The multifrequency SEDs for the sample of EGRET sources.

The association of some of the unidentified EGRET sources with Seyfert galaxies/LINERS poses a possibility of non-aligned AGN and radio galaxies constituting a new class of gamma-ray sources. Before discussing the modeling of the SEDs presented above, a brief discussion of the gamma-ray properties of some radio galaxies are presented to illustrate that these sources possess the required energetics to produce measurable $\gamma$-ray emission in the EGRET and Fermi-LAT energy domain

\section{Non-Aligned AGN: A New Class of Gamma-Ray Source}

At High Energies (HE; $\left.E_{\gamma}>100 \mathrm{MeV}\right)$ Fermi-LAT reported about ten misaligned radio galaxies (Abdo et al., 2010b,c; Rieger, 2012a). At Very High Energies (VHE; $\left.E_{\gamma}>100 \mathrm{GeV}\right)$, four radio galaxies have been detected, Cen $\mathrm{A}(d \approx 3.8 \mathrm{Mpc}), \mathrm{M} 87(d \approx 16.7 \mathrm{Mpc})$ and the Perseus Cluster $(d \approx 77 \mathrm{Mpc} ; \mathrm{z} \approx 0.018)$ sources NGC 1275 and IC 310 (e.g. Rieger, 2012a). Cen A was the only non-blazar detected at $\mathrm{MeV}$ to $\mathrm{GeV}$ energies by CGRO (see Steinle, 2010 for a review). The HE emission from Cen A reported by Fermi-LAT seems to come from both the extended radio lobes and the core region (Abdo et al., 2010b,c). The detected HE gamma-rays from the extended lobe regions suggests that particle acceleration up to VHEs occurs in the disrupted jet region (Bordas, 2012). The reported $\mathrm{TeV}$ emission from Cen A (Aharonian et al., 2009) provided further evidence of a very effective particle accelerator in Cen A. The spectrum up to $5 \mathrm{TeV}$ is consistent with a power-law with a photon index $\sim 2.7 \pm 0.5$, with no apparent variability. The $\mathrm{HE}\left(E_{\gamma}>0.2 \mathrm{GeV}\right)$ emission in Cen $\mathrm{A}$ is explained in terms of inverse Compton (IC) upscattering of the Cosmic Microwave Background (CMB) photons $\left(E_{\mathrm{ph}}=8 \times 10^{-4} \mathrm{eV}\right)$ and infrared (IR) background photons by relativistic electrons with Lorentz factors $\gamma_{\mathrm{e}}=6 \times 10^{5}$, in a jet with bulk flow velocities between $\beta_{\Gamma} \sim 0.1-0.5$. Another peculiar aspect of Cen $\mathrm{A}$ is a rather low, sub-Eddington inferred accretion rate, $\dot{m} \sim 10^{-3} \dot{m}_{\text {Edd }}$, resulting in a rather low bolometric luminosity $L_{\mathrm{b}} \sim 10^{43} \mathrm{erg} \mathrm{s}^{-1}$ (Whysong \& Antonucci, 2004). The inferred equipartition magnetic field in the radio lobes is $\mathrm{B} \approx 9 \mu \mathrm{G}$ (Rieger, 2012b), with the equipartition magnetic field near the black hole $(\mathrm{BH})$ ranging between $B \sim 10^{3}-10^{4} \mathrm{G}$ (Rieger, $2012 \mathrm{~b}$ ). The detection of non-aligned AGN in the HE and VHE regime poses interesting theoretical challenges regarding particle acceleration and associated gammaray emission in the jets of AGN. For example, Cen A shows that the radio lobes of radio galaxies may possess the required energetics to accelerate electrons to VHEs producing the HE gamma-rays through IC upscattering the CMB photons, even though the bulk flow Lorentz factor of the jet is fairly low. The detection of $\mathrm{TeV}$ gamma-rays is explained in terms of $\gamma_{\mathrm{e}} \sim 10^{7}$ electrons up-scattering disc photons to the $\mathrm{TeV}$ domain (Rieger \& Aharonian, 2009). The nuclear SED of Cen A, based on non-simultaneous data, shows two peaks, one around $\sim 10^{13} \mathrm{~Hz}$ and another around $0.1 \mathrm{MeV}$ (e.g. Chiaberge et al., 2001; Abdo et al., 2010c). The SED below a few $\mathrm{GeV}$ seems to be satisfactorily explained by a one-zone Synchrotron self-Compton (SSC) model (Chiaberge et al., 2001) but the same approach fail to account for the $\mathrm{TeV}$ emission observed by H.E.S.S. (Abdo et al., 2010c). 
The discussion presented above underlines the fact that normal, non-aligned AGN do possess the required energetics to accelerate leptons to $\mathrm{VHE}$ energies. The production of sub-GeV gamma-rays through a SSC process in the nuclear region, combined with the HE emission in the radio lobes and $\mathrm{TeV}$ emission in the inner disc region close to the black hole $(\mathrm{BH})$, presents a new paradigm in particle acceleration and gamma-ray production in AGN. With this in mind, the SED of the Fermi-LAT counterparts of the unidentified EGRET sources were modelled. This is a first attempt to explain the SED of these sources within the framework of a Synchrotron self-Compton (SSC) and External Compton (EC) model. These sources are all treated as misaligned AGN, with a fixed inclination of $\theta_{\text {jet }} \approx 60^{\circ}$ between jet and observer.

\section{$6 \quad$ SED Modelling}

The multi-wavelength data from radio to gamma-rays have been combined to create the SED over more than 15 decades in energy (Fig. 7). The EGRET and Fermi$L A T$ gamma-ray data are evaluated with the theoretical framework of a single zone Synchrotron self-Compton (SSC) (e.g. Katarczynski, Sol \& Kus, 2001) and External Compton (EC) model (e.g. Moderski et al., 2003; Sikora, Begelman \& Rees, 1994), i.e. where relativistic jet electrons up-scatter infrared (IR) photons from the disc torus and optical photons from the broad emission line (BEL) regions to high energies. The model parameters for the SSC and EC models are presented in Table 1 (SSC) and Table 2 (EC). The corresponding SED model fits are presented in Fig. 8.

Table 1: Parameters related to SSC models for the respective sources.

\begin{tabular}{lllllll}
\hline & Source & & 3EG J0724-4713 & 3EG J1659-6251 & 3EG J1709-0828 & 3EG J1813-6419 \\
& Parameter & Units & & & & \\
\hline \hline 1 & radius & $(\mathrm{m})$ & $1.5 \mathrm{E}+14$ & $1.0 \mathrm{E}+14$ & $1.0 \mathrm{E}+14$ & $1.0 \mathrm{E}+14$ \\
2 & $\mathrm{~B}$ & $(\mathrm{~T})$ & $7.0 \mathrm{E}-04$ & $7.5 \mathrm{E}-04$ & $2.5 \mathrm{E}-04$ & $2.5 \mathrm{E}-04$ \\
3 & $p_{1}$ & & -2 & -2 & -2 & -2 \\
4 & $p_{2}$ & & -3 & -2.6 & -2.6 & -2.6 \\
5 & $\gamma_{\max }$ & & $1.6 \mathrm{E}+03$ & $6.2 \mathrm{E}+02$ & $1.6 \mathrm{E}+03$ & $2.0 \mathrm{E}+03$
\end{tabular}

Table 2: Parameters related to the EC models for the respective sources.

\begin{tabular}{llllllll}
\hline Source & $\begin{array}{l}\mathrm{A}_{\text {obs }} \\
(1)\end{array}$ & $\begin{array}{l}\gamma_{\mathrm{br}} \\
(3)\end{array}$ & $\begin{array}{l}K_{\mathrm{e}} \\
(4)\end{array}$ & $\begin{array}{l}\nu_{\mathrm{BEL}} \\
(5)\end{array}$ & $\begin{array}{l}\alpha_{\mathrm{BEL}} \\
(6)\end{array}$ & $\begin{array}{l}\nu_{I R} \\
(7)\end{array}$ \\
\hline \hline 3EG J0724-4713 & $1.0 \mathrm{E}+17$ & 1 & $2.0 \mathrm{E}+03$ & $4.0 \mathrm{E}+55$ & 10 & -1 & 0.1 \\
3EG J1300-4406 (BEL) & $1.0 \mathrm{E}+17$ & 1 & $2.0 \mathrm{E}+04$ & $6.0 \mathrm{E}+53$ & 1 & -1 & 0.1 \\
3EG J1300-4406 (IR) & $1.0 \mathrm{E}+17$ & 1 & $3.0 \mathrm{E}+03$ & $4.0 \mathrm{E}+54$ & 1 & -1 & 0.1 \\
3EG J1659-6251 (BEL) & $1.0 \mathrm{E}+17$ & 1 & $2.0 \mathrm{E}+03$ & $6.0 \mathrm{E}+54$ & 10 & -1 & 0.1 \\
3EG J1659-6251 (IR) & $1.0 \mathrm{E}+17$ & 1 & $2.0 \mathrm{E}+03$ & $3.0 \mathrm{E}+55$ & 10 & -0.5 & 0.1 \\
3EG J1709-0828 & $1.0 \mathrm{E}+17$ & 1 & $2.0 \mathrm{E}+03$ & $3.0 \mathrm{E}+55$ & 10 & -1 & 0.1 \\
3EG J1800-0146 & $2.5 \mathrm{E}+16$ & 1 & $5.0 \mathrm{E}+02$ & $3.0 \mathrm{E}+55$ & 1 & -1 & 0.1 \\
3EG J1813-6419 & $1.0 \mathrm{E}+17$ & 1 & $2.4 \mathrm{E}+03$ & $1.3 \mathrm{E}+55$ & 10 & -1 & 0.1
\end{tabular}

(1): Radius of the emitting region (in $\mathrm{cm}$ ), (2): Viewing angle (in radians), (3): Electron Lorentz factor at spectral break, (4): Electron normalization constant, (5): Characteristic frequency of BEL (in eV), (6): BEL distribution photon index, (7): Radiation characteristic frequency of IR (in $\mathrm{eV}$ ). 


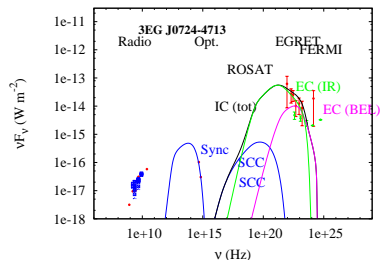

a.) $3 E G$ J0724-4713

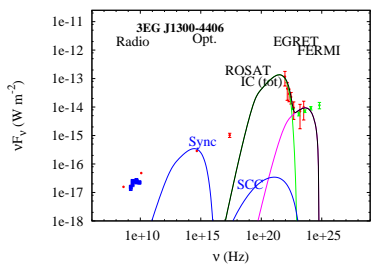

c.) $3 E G J 1300-4406$

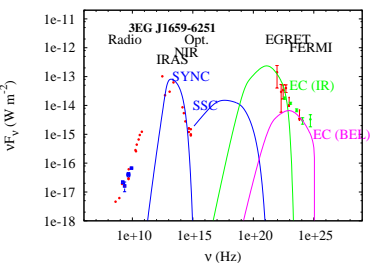

b.) $3 E G J 1659-625$

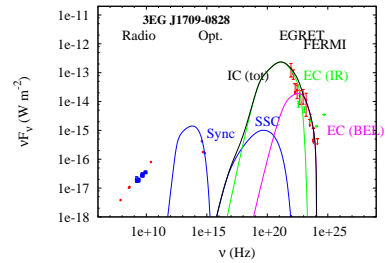

d.) $3 E G$ J1709-0828

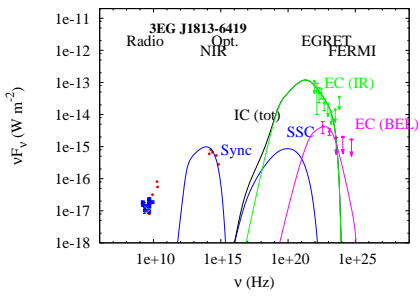

e.) 3EG J1813-6419

Figure 8: The modeled SEDs for the various EGRET/Fermi-LAT sources.

From these results (see Fig. 8) it can be seen that the EGRET and Fermi-LAT data are consistent with an EC model, i.e. the IC upscattering of IR photons from the disc and UV/optical photons from the line emitting clouds (Broad Line Regions) to the EGRET and Fermi-LAT energy domain. In all the models the electron Lorentz factors were $\gamma_{\mathrm{e}} \sim$ few $\times 10^{3}$, rather modest in comparison with the Lorentz factors required to explain the $\gamma$-ray emission from, for example, Cen A.

\section{Conclusions}

We report the discovery of 13 flat spectrum extragalactic sources within the error boxes of some high galactic latitude, unidentified EGRET sources. Five of these EGRET sources have been detected with Fermi-LAT within the first 11 months of operation. In all cases the EGRET and Fermi-LAT gamma-ray emission could be successfully explained in terms of the IC upscattering of BEL photons, as well as IR photons from the disc, to EGRET and Fermi-LAT energies. The adopted electron energy is of the order of $\gamma_{\mathrm{e}} \sim$ few $\times 10^{3}$, which is rather moderate compared to the electron energies of $\gamma_{\mathrm{e}} \sim 10^{6}-10^{7}$, inferred from the $\mathrm{HE}$ and $\mathrm{TeV}$ emission from Cen A. These preliminary results definitely confirm that Seyfert and radio galaxies could be associated with a significant fraction of the still unidentified extragalactic EGRET and Fermi-LAT sources.

\section{Acknowledgement}

The authors thank the organisers for the invitation to present this work at this conference. Some of the observations reported in this paper were performed by the Southern African Large Telescope (SALT). The SALT observations were performed under the Proposal: 20121-RSA-003 (PI: B van Soelen). The authors thank Dr. Petri Vaisanen from the SALT science team for his guidance with the data reduction.

\section{References}

[1] Abdo, A.A., et al.(FERMI): 2010a, ApJ, 710, 1271 doi:10.1088/0004-637X/710/2/1271

[2] Abdo, A.A., et al. (FERMI): 2010b, Science, 328, 725 doi:10.1126/science. 1184656

[3] Abdo, A.A., et al. (FERMI): 2010c, ApJ, 719, 1433 doi:10.1088/0004-637X/719/2/1433

[4] Aharonian, F.A., et al. (H.E.S.S.): 2009, ApJ, 695, L40 doi:10. 1088/0004-637X/695/1/L40

[5] Bordas, P.: 2012 in Multifrequency Behaviour of High Energy Cosmic Sources, Vulcano (May 2328, 2011), MEMORIE S.A.It, Vol 83 (1), 87 (eds. F. Giovanelli \& L. Sabau-Graziati)

[6] Caccianiga, A., della Ceca, R., Gioi, I.M., Maccacaro, T. \& Wolter, T.: 1999, ApJ, 513, 51

[7] Chiaberge, M. et al.: 2001, MNRAS, 324, 33 doi:10.1046/j.1365-8711.2001.04642. $\mathrm{x}$

[8] Fan, J-H.: 2005, Chin. J. Astron. Astrophys. Suppl., 5, 213 doi:10.1088/1009-9271/5/S1/213

[9] Hartman, R., et al.: 1999, APJS, 123, 79

[10] Katarzynski, K., Sol, H. \& Kus, A.: 2001, A\&A, 367,809

[11] Mattox, J., et al.: 1997, ApJ, 476, 692

[12] Meintjes, P.J. \& Nkundabakura, P.: 2012, in High Energy Gamma-Ray Astronomy, Proc of the 5th International High Energy Gamma-Ray Astronomy meeting, Heidelberg (Germany), AIP Conference proceedings, Vol. 1505, p. 554 (eds. F.A. Aharonian, W. Hoffman \& F. Rieger), Publisher, Melville (New York) 
[13] Moderski, R., et al.: 2003, A\&A, 406, 855

[14] Nkundabakura, P.J.: P. \& Meintjes, MNRAS, 427 doi:10.1111/j.1365-2966.2012.21953.x

[15] Nolan, P.L., et al. (FERMI): 2011, ApJ Suppl., 199, 31 doi:10.1088/0067-0049/199/2/31

[16] Rieger, F. \& Aharonian, F.A.: 2009, A\&A, 506, L41

[17] Rieger, F.: 2012a, in High Energy Gamma-Ray Astronomy, Proc of the 5th International High Energy Gamma-Ray Astronomy meeting, Heidelberg (Germany), AIP Conference proceedings, Vol. 1505, p. 80 (eds. F.A. Aharonian, W. Hoffman \& F. Rieger), Publisher, Melville (New York)
[18] Rieger, F.: 2012b, in Multifrequency Behaviour of High Energy Cosmic Sources, Vulcano (May 23-28, 2011), MEMORIE S.A.It, Vol 83 (1), 127 (eds. F. Giovanelli \& L. Sabau-Graziati)

[19] Sikora, M., Begelman, M.C. \& Rees, M.J.: 1994, ApJ, 421, 153

[20] Sowards-Emmerd, D., Romani, R.W. \& Michelson, P.F.: 2003, ApJ, 590, 109

[21] Steinle, H.: 2010, PASA, 27, 431

[22] Whysong, D. \& Antonucci, R.: 2004, ApJ, 602, 116 